\title{
Analyse modale d'une structure industrielle avec prise en compte du couplage fluide/structure
}

\author{
Jean-François Sigrist, ${ }^{1, a}$, Christian Lainé ${ }^{1}$ et Bernard Peseux ${ }^{2}$ \\ 1 DCN Propulsion, Service Scientifique et Technique, 44620 La Montagne, France \\ 2 École Centrale de Nantes, Laboratoire Mécanique Matériaux, 1 rue de La Noë, 44321 Nantes Cedex, France
}

Reçu le 15 décembre 2003, accepté le 2 juillet 2004

\begin{abstract}
Résumé - Nous présentons une analyse modale d'une structure industrielle couplée avec un fluide, en utilisant les techniques numériques de calculs couplés fluide/structure. Compte tenu de la nature axisymétrique de la géométrie et de la nature non axisymétrique des équations de couplage, la modélisation du problème est réalisée au moyen d'éléments finis axisymétriques développés en série de Fourier. Un code de calcul est implanté dans Matlab pour permettre l'analyse modale de la structure. Différentes formulations du problème sont comparées; les résultats de calcul Matlab sont comparés avec les résultats de calculs obtenus avec le code généraliste Ansys. Les développements mis en œuvre pour cet exemple seront à terme intégrés dans le code Ansys pour l'étude de problèmes couplés en pression/déplacement avec développement en série de Fourier.
\end{abstract}

Mots clés : Analyse modale / interactions fluide/structure / éléments finis axisymétriques en série de Fourier / formulations symétrique et non symétrique

\begin{abstract}
Modal analysis of an industrial structure with fluid-structure interaction. This paper gives an example of numerical methods with finite element coupling for the numerical study of an industrial fluid/structure coupled problem. The problem is solved by coupling a finite element discretization of both fluid and structure domain. The coupled system is described in terms of pressure and displacement for the fluid and structure problem. The unknown degrees of freedom are expanded in a Fourier serie. A numerical code is developed in Matlab in order to perform the modal analysis of the coupled problem. Numerical calculations are performed and compared with the Ansys code in order to validate our numerical developments.
\end{abstract}

Key words: Modal Analysis / fluid-structure inetraction / harmonic axisymmetric finite elements / symmetric and non-symmetric formulations

\section{Introduction}

La simulation numérique de problèmes couplés fluide/structure a fait l'objet de nombreuses études numériques qui ont permis le développement de méthodes de calcul, consistant à réaliser un couplage entre une discrétisation de type éléments finis du domaine structure et une discrétisation de type équation intégrale ou éléments finis du problème fluide $[1,2]$. Ces techniques de calcul sont développées dans des laboratoires de recherche, et appliquées par exemple pour réaliser l'étude de structures immergées, avec prise en compte ou non de phénomènes de surface libre dans le fluide [3-10], mais leur utilisation courante en bureau d'étude est cependant assez rare dans le cadre de projets industriels $[11,12]$.

\footnotetext{
${ }^{a}$ Auteur correspondant : jean-francois.sigrist@dcn.fr
}

Le présent article se propose d'illustrer une application de ces techniques de calcul à l'étude dynamique d'une structure représentative de l'ensemble panier et cuve d'un réacteur de propulsion navale, avec prise en compte du couplage fluide/structure.

Le modèle simplifié de la structure étudiée est représenté par la figure 1. On adopte une modélisation axisymétrique de l'ensemble et on distingue quatre principaux éléments :

- le corps du panier (1) : il s'agit de la partie supérieure de la structure intérieure du réacteur, qui contient les éléments combustibles. La géométrie est essentiellement cylindrique. Les caractéristiques géométriques et physiques sont notées $E_{1}$ (module d'Young), $\rho_{1}$ (masse volumique), $\nu_{1}$ (coefficient de Poisson) et $e_{1}$ (épaisseur); 


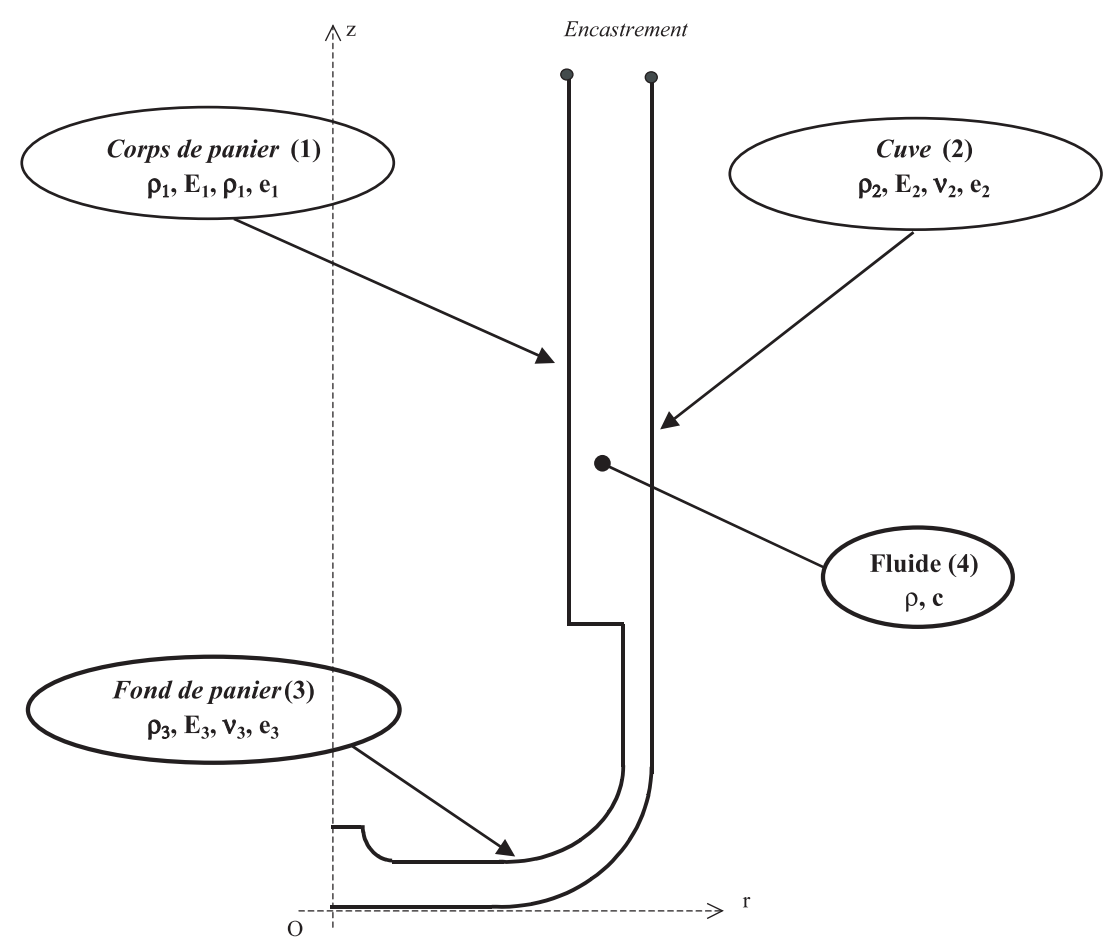

Fig. 1. Géométrie industrielle étudiée. Représentation axisymétrique de l'ensemble panier et cuve d'un réacteur de propulsion navale.

- le fond du panier (2) : il s'agit de la partie inférieure de la structure intérieure du réacteur. La géométrie est plus complexe et fait apparaître deux parties sphériques. Les grandeurs caractéristiques sont notées $E_{2}, \rho_{2}, \nu_{2}$ et $e_{2}$;

- la cuve (3) : il s'agit de l'enveloppe externe du réacteur. Elle est modélisée en première analyse d'une partie cylindrique et d'une partie sphérique. Les grandeurs caractéristiques sont notées $E_{3}, \rho_{3}, \nu_{3}$ et $e_{3}$;

- le fluide primaire (4) : il est contenu entre le panier et la cuve. On note $\rho_{\mathrm{F}}$ sa masse volumique et $c$ la célérité des ondes sonores dans ce milieu.

Parmi la grande variété de modélisations géométriques et de représentations des inconnues du problème couplé, nous privilégions une approche compatible avec les exigences d'analyse en bureau d'étude : simplicité du modèle géométrique, interprétation aisée des modes propres.

L'analyse est basée sur une discrétisation de type éléments finis pour la structure, couplée avec une discrétisation de type éléments finis pour le fluide. La modélisation adopte une formulation :

- en déplacement pour la structure, les inconnues du problème structure sont les déplacements, notés $u_{r}$, $u_{\theta}$ et $u_{z}$ en coordonnées cylindriques (approche lagrangienne);

- en pression pour le fluide, l'inconnue du problème fluide est le champ de pression $p$ (approche eulérienne).

Compte tenu de l'hypothèse de modélisation axisymétrique de la géométrie et de la nature non axisymétrique de la sollicitation dynamique appliquée sur la structure, les inconnues du problème couplé en série de Fourier sont développées selon la relation $[13,14]$ :

$$
\begin{array}{r}
\left\{\begin{array}{l}
u_{r} \\
u_{\theta} \\
u_{z} \\
p
\end{array}\right\}=\left\{\begin{array}{l}
u_{r}^{0} \\
u_{\theta}^{0} \\
u_{z}^{0} \\
p^{0}
\end{array}\right\}+\sum_{n=1}^{n=+\infty}\left\{\begin{array}{l}
u_{r}^{n} \cos (n \theta) \\
u_{\theta}^{n} \sin (n \theta) \\
u_{z}^{n} \cos (n \theta) \\
p^{n} \cos (n \theta)
\end{array}\right\} \\
+\sum_{m=1}^{m=+\infty}\left\{\begin{array}{l}
u_{r}^{m} \sin (m \theta) \\
-u_{\theta}^{m} \cos (m \theta) \\
u_{z}^{m} \sin (m \theta) \\
p^{m} \sin (m \theta)
\end{array}\right\}
\end{array}
$$

Ce développement fait apparaître des termes axisymétriques (ordre 0), des termes symétriques (ordre $n \geq$ 1 ) et des termes antisymétriques (ordre $m \geq 1$ ). La sollicitation dynamique appliquée correspond à un chargement dans une direction transverse à la structure de révolution, nous nous s'intéressons donc à la détermination des modes symétriques d'ordre 1 (i.e. en $\cos \theta, n=1$ ).

La discrétisation du problème couplé est réalisée dans un code développé dans Matlab [15], utilisant un élément coque axisymétrique ${ }^{1}$ et un élément acoustique

\footnotetext{
${ }^{1}$ Compte tenu des épaisseurs de la structure industrielle, la modélisation par éléments coque atteint ses limites. L'objet du présent travail étant de mettre en évidence le couplage des modes de structure par le fluide, l'utilisation d'un modèle de coque élastique reste cependant justifiée dans ce contexte.
} 
axisymétrique. Les calculs seront comparés avec des calculs éléments finis réalisés avec le code commercial Ansys $^{2}$ [16], utilisant deux types de modèles :

- modèle bidimensionnel axisymétrique mettant en œuvre des éléments finis axisymétriques développés en série de Fourier et formulés en déplacement;

- modèle tridimensionnel mettant en œuvre des éléments finis volumiques formulés en pression/ déplacement.

Le choix d'une représentation en pression/ déplacement du problème couplé, avec développement des inconnues en série de Fourier, présente les avantages suivants et répond aux exigences d'une analyse pour application en bureau d'étude :

- représentation simple du problème fluide par la variable pression;

- non singularité des matrices de masse et de raideur du problème fluide pour les harmoniques $n \geq 1$ et $m \geq 1$;

- absence de modes propres non physique pouvant parasiter l'analyse modale.

L'analyse modale conduite sur le problème industriel est réalisée en considérant la structure élastique (panier et cuve) seule, le fluide acoustique seul et enfin le problème couplé élasto-acoustique. Dans ce dernier cas, différentes formulations sont étudiées.

\section{1 Étude de la structure dans le vide}

Nous commençons l'étude par une analyse modale sur chaque élément de la structure (panier et cuve) dans le vide. La discrétisation du problème utilise des éléments coque à deux nœuds et quatre degrés de liberté par nœud (voir Fig. 2).

Le champ de déformations est donné par le modèle de Sanders-Koiters, qui permet de décrire le comportement en flexion de la coque élastique [18]. La relation déplacements/déformations est alors la suivante :

$$
\{\varepsilon\}=\left\{\begin{array}{c}
-\frac{\partial u_{z}}{\partial z} \\
-\frac{1}{r} \frac{\partial u_{\theta}}{\partial \theta}+\frac{u_{r}}{r} \\
-\frac{1}{r} \frac{\partial u_{z}}{\partial \theta}+\frac{\partial u_{r}}{\partial z} \\
-\frac{\partial^{2} u_{r}}{\partial z^{2}} \\
\frac{1}{r^{2}}\left(\frac{\partial^{2} u_{r}}{\partial \theta^{2}}+\frac{\partial u_{\theta}}{\partial \theta}\right) \\
\frac{2}{r}\left(-\frac{\partial^{2} u_{r}}{\partial z \partial \theta}-\frac{3}{4} \frac{\partial u_{\theta}}{\partial z}+\frac{1}{4 r^{2}} \frac{\partial u_{z}}{\partial \theta}\right)
\end{array}\right\}
$$

${ }^{2}$ Un précédent travail a permis de valider, par comparaison calcul/essai, l'utilisation du code Ansys pour l'analyse modale de structures élastiques couplées avec un fluide acoustique [17].

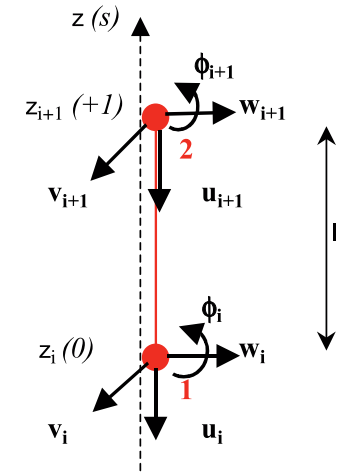

Élément de référence

$$
\Omega_{\mathrm{S}}^{\mathrm{e}(\mathrm{s}, \theta)}
$$

Fig. 2. Élément fini coque.

Tableau 1. Premières fréquences propres du panier dans le vide.

\begin{tabular}{rrrr}
\hline Fréquence $(\mathrm{Hz})$ & Calcul Ansys & Calcul Matlab & $\varepsilon(\%)$ \\
\hline$f_{1}$ & 44,69 & 44,70 & 0,02 \\
$f_{2}$ & 140,67 & 140,77 & 0,07 \\
$f_{3}$ & 245,71 & 245,72 & 0,00 \\
$f_{4}$ & 284,25 & 284,62 & 0,13 \\
$f_{5}$ & 322,1 & 322,80 & 0,21 \\
\hline
\end{tabular}

Tableau 2. Premières fréquences propres de la cuve dans le vide.

\begin{tabular}{rrrr}
\hline Fréquence $(\mathrm{Hz})$ & Calcul Ansys & Calcul Matlab & $\varepsilon(\%)$ \\
\hline$f_{1}$ & 78,71 & 78,708 & $-0,01$ \\
$f_{2}$ & 260,65 & 260,92 & 0,10 \\
$f_{3}$ & 431,58 & 424,88 & $-1,55$ \\
$f_{4}$ & 480,22 & 480,64 & 0,09 \\
$f_{5}$ & 555,87 & 553,28 & 0,47 \\
\hline
\end{tabular}

L'analyse modale de la structure consiste à résoudre le problème aux valeurs propres symétrique suivant :

$$
\left(\mathbf{K}_{\mathrm{S}}-\omega^{2} \mathbf{M}_{\mathrm{S}}\right) \times \mathbf{U}(\omega)=\mathbf{0}
$$

avec $\mathbf{K}_{\mathrm{S}}$ et $\mathbf{M}_{\mathrm{S}}$ les matrices du système structure. Le problème aux valeurs propres est symétrique, nous utilisons l'algorithme de Cholesky $[19,20]$ pour extraire les modes propres du système.

Les premières fréquences propres du panier et de la cuve, calculées avec le code développé dans Matlab, sont données dans les tableaux 1 et 2 et comparées avec les fréquences propres calculées avec Ansys, en utilisant un modèle de coque élastique axisymétrique en série de Fourier (éléments shell61 [16]). Les déformées modales correspondantes dans le plan $(r, z)$ sont données par la figure 3 ; on considère les modes symétriques d'harmonique $n=1$, la sollicitation dynamique sur la structure étant en $\cos (\theta)$. Les résultats donnés par le code développé dans Matlab sont identiques aux résultats de calcul Ansys, à la fois en termes de valeurs des fréquences propres que de déformées modales.

La figure 4 donne les courbes de convergence des premières fréquences propres du panier et de la cuve pour 

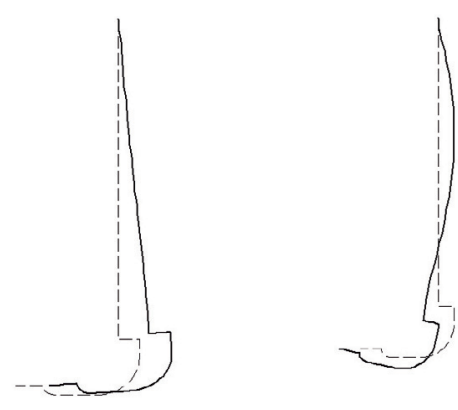

(a) Panier

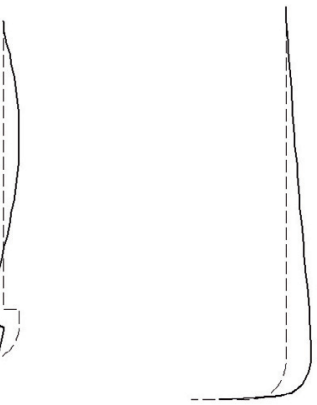

(b) Cuve

$$
f_{1}=44,7 \mathrm{~Hz} \quad f_{2}=140,8 \mathrm{~Hz} \quad f_{1}=78,7 \mathrm{~Hz} \quad f_{1}=260,9 \mathrm{~Hz}
$$

Fig. 3. Premiers modes propres d'ordre $n=1$ du panier (a) et de la cuve (b) dans le vide obtenus avec le code de calcul développé dans Matlab.

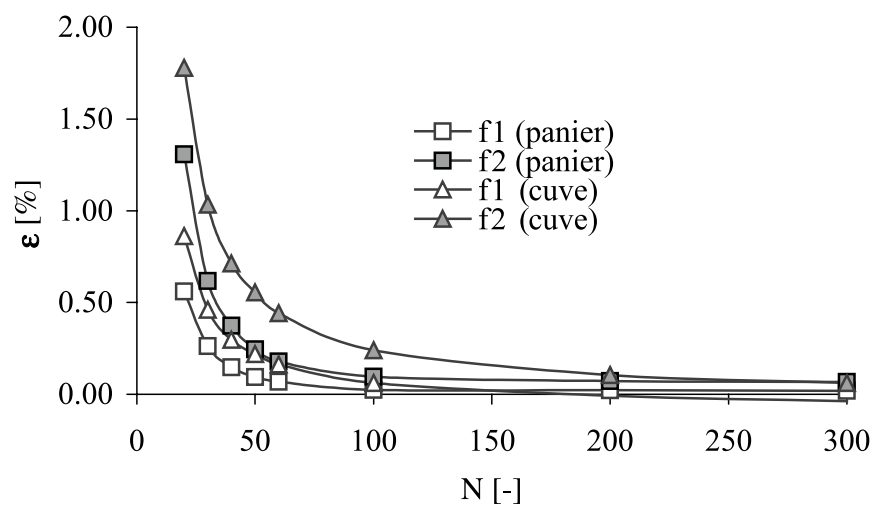

Fig. 4. Courbes de convergence des premières fréquences propres du panier et de la cuve dans le vide.

un nombre $N$ d'éléments, soit un nombre total de degrés de liberté de $4 N$ (en ordonnée, on porte $\varepsilon$ l'écart relatif entre les résultats du calcul Matlab et le résultat du calcul de référence avec Ansys avec les éléments shell61). La convergence est obtenue assez rapidement pour les modèles étudiés, ce qui permettra de travailler avec des modèles de taille raisonnable dans le cas de l'analyse modale du système avec couplage fluide/structure.

\section{2 Étude de la cavité acoustique seule}

Nous réalisons ensuite une analyse modale de la cavité fluide délimitée par le panier et la cuve, considérés comme rigides. La discrétisation du domaine fluide utilise des éléments finis linéaires à quatre nœuds et un degré de liberté par nœud (voir Fig. 5).

Le maillage de l'ensemble est visualisé par la figure 6 .

L'analyse modale de la cavité acoustique consiste à résoudre le problème aux valeurs propres symétrique suivant :

$$
\left(\mathbf{K}_{\mathrm{F}}-\omega^{2} \mathbf{M}_{\mathrm{F}}\right) \times \mathbf{P}(\omega)=\mathbf{0}
$$

avec $\mathbf{K}_{\mathrm{F}}$ et $\mathbf{M}_{\mathrm{F}}$ les matrices du système fluide. Nous utilisons les mêmes méthodes d'extraction de valeurs propres que pour le problème structure.

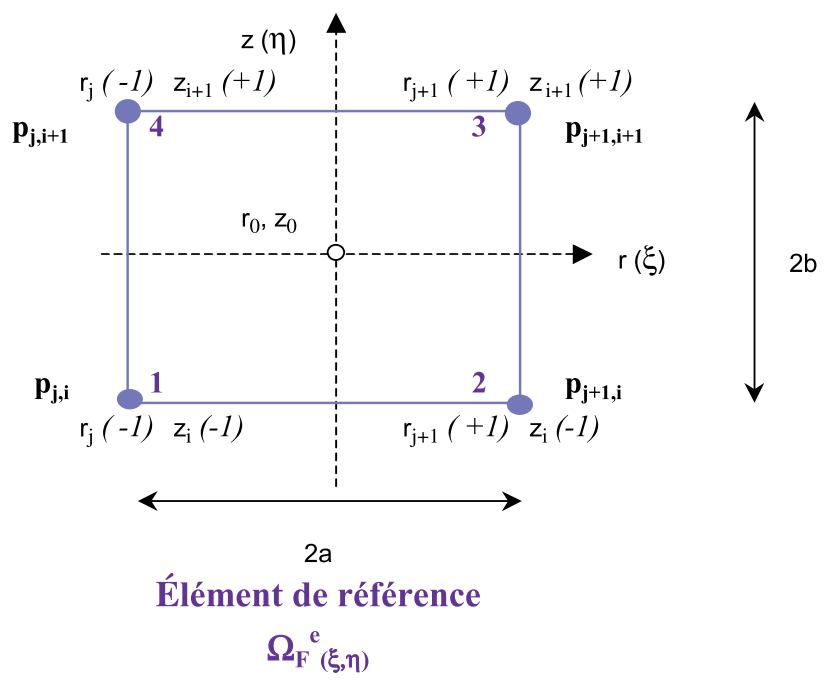

Fig. 5. Élément fini fluide.

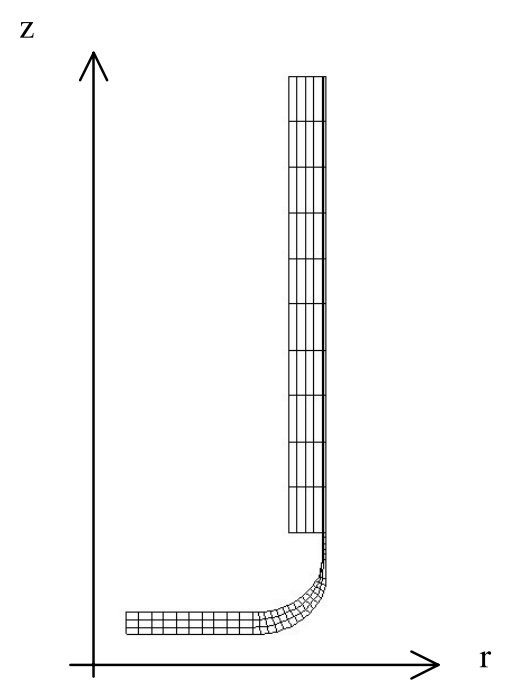

Fig. 6. Maillage de la cavité acoustique délimitée par le panier et la cuve dans Matlab. 
Tableau 3. Premières fréquences propres de la cavité acoustique délimitée par le panier et la cuve.

\begin{tabular}{rrrr}
\hline Fréquence $(\mathrm{Hz})$ & Calcul Ansys & Calcul Matlab & $\varepsilon(\%)$ \\
\hline$f_{1}$ & 224,58 & 224,57 & 0,00 \\
$f_{2}$ & 307,96 & 307,04 & $-0,30$ \\
$f_{3}$ & 419,06 & 406,12 & $-3,09$ \\
$f_{4}$ & 479,80 & 482,52 & 0,57 \\
$f_{5}$ & 669,69 & 669,87 & 0,93 \\
\hline
\end{tabular}

Les premières fréquences propres de la cavité (pour l'harmonique $n=1$ ), calculées avec le code développé dans Matlab, sont données dans le tableau 3 et comparées avec les fréquences propres calculées avec Ansys, utilisant les éléments fluides acoustiques formulés en déplacement ${ }^{3}$ et développés en série de Fourier (éléments fluid81 du code [16]). Les formes modales correspondantes (représentées en iso-valeurs de pression) dans le plan $(r, z)$ sont données par la figure 7 . Les résultats donnés par le code développé dans Matlab sont identiques aux résultats de calcul Ansys, à la fois en termes de valeurs des fréquences propres que de déformées modales. Le problème fluide comporte environ $N=350$ degrés de liberté.

\section{3 Étude de la structure couplée avec le fluide}

\subsection{Approche simplifiée}

L'approche simplifiée du problème consiste à utiliser un modèle de masse d'eau reportée pour prendre en compte la présence du fluide : la masse d'eau présente entre le panier et la cuve est reportée pour moitié entre les deux structures, ce qui permet de respecter le bilan masse total de l'ensemble structure et fluide. Cet artifice de calcul ne permet pas de bien décrire les effets du fluide sur la structure : l'utilisation du modèle de masse d'eau reportée conduit à sous-estimer très largement dans ce cas l'influence du fluide sur la structure, comme le montrent les résultats du tableau 4. Compte tenu du confinement, la masse d'eau ajoutée (qui traduit l'effet physique du fluide sur la structure) est nettement plus importante que la masse d'eau reportée : la baisse de fréquence propre est moins importante avec le modèle de masse d'eau reportée qu'avec le modèle de masse d'eau ajoutée [22]. D'autre part, les effets de couplage des modes de cuve et de panier par le fluide ne sont pas pris en compte dans un modèle de masse d'eau reportée.

\footnotetext{
${ }^{3}$ Dans Ansys, les éléments fluides à formulation en déplacement sont obtenus par modification des éléments solides. La différence entre les éléments fluides et solides est principalement dans la loi de comportement : la raideur en cisaillement est proche de zéro et les termes associés aux contraintes sont ramenés à la valeur du module du fluide. On obtient ainsi un élément qui ne peut reprendre le cisaillement, mais dont les composantes de contrainte normale sont proches de la pression instantanée $[16,21]$.
}

Tableau 4. Fréquences propres du système panier et cuve. Comparaison entre un calcul sans eau, avec masse d'eau reportée et avec masse d'eau ajoutée (calcul couplé avec le code Ansys par couplage éléments finis/éléments finis en formulation déplacement/déplacement avec représentation des inconnues en série de Fourier).

\begin{tabular}{rrrr}
\hline Fréquence $(\mathrm{Hz})$ & Sans eau & $\begin{array}{r}\text { Masse d'eau } \\
\text { reportée }\end{array}$ & $\begin{array}{r}\text { Masse d'eau } \\
\text { ajoutée }\end{array}$ \\
\hline$f_{1}$ & 44,70 & 43,38 & 26,03 \\
$f_{2}$ & 78,71 & 75,55 & 65,52 \\
$f_{3}$ & 140,77 & 136,56 & 102,94 \\
$f_{4}$ & 245,71 & 238,27 & 162,30 \\
$f_{5}$ & 260,65 & 250,19 & 180,32 \\
\hline
\end{tabular}

Ceci met en évidence l'intérêt de mettre en œuvre un calcul couplé fluide/structure pour l'analyse modale de la structure industrielle.

Les résultats des calculs couplés sont obtenus avec le code Ansys, dans une formulation déplacement/déplacement. Cette formulation possède l'inconvénient de générer des modes non physiques, qui peuvent perturber l'analyse modale : ces modes correspondent aux mouvements de cisaillement du fluide sans changement de volume, la structure restant fixe. On lui préfère, pour une application en bureau d'étude, une formulation en pression/déplacement (non symétrique) ou en pression/potentiel des déplacements/déplacement (symétrique), qui ne présente pas ces inconvénients [21]. Cependant, le code Ansys ne permet pas de traiter, pour ce type de formulation, des problèmes axisymétriques avec un développement des inconnues en série de Fourier [16]. Nous développons donc un code dans le progiciel Matlab permettant de réaliser l'analyse modale de la structure étudiée, en utilisant les éléments finis présentés aux sections précédentes.

\subsection{Approche « couplage fort », formulation non symétrique}

La technique de couplage éléments finis/éléments finis consiste à discrétiser les termes d'échanges entre le fluide et la structure; on calcule ainsi une matrice d'interaction fluide/structure en couplant les degrés de liberté en déplacement de la structure et le degré de liberté pression du fluide (Fig. 8).

La matrice de couplage est calculée par assemblage des matrices élémentaires de couplage $\mathbf{r}_{i}$, pour un couplage défini par la figure 8 . Chaque matrice élémentaire $\mathbf{r}_{i}$ est calculée à partir des fonctions de forme des deux éléments, selon la relation :

$$
\mathbf{r}_{i}=\int_{z_{i}}^{z_{i+1}}\left\{\boldsymbol{N}_{\mathrm{F}}(z)\right\}\left\langle\boldsymbol{n}_{i}\right\rangle\left[\mathbf{N}_{\mathrm{S}}(z)\right] \mathrm{d} z
$$

avec $\left\{\boldsymbol{N}_{\mathrm{F}}\right\}$ le vecteur des fonctions de forme de l'élément fluide, $\left[\mathbf{N}_{\mathrm{S}}\right]$ la matrice des fonctions de forme de l'élément structure et $\left\langle\boldsymbol{n}_{i}\right\rangle$ le vecteur des composantes de la normale locale sortante du milieu fluide. 


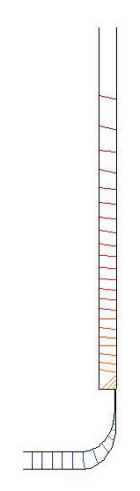

$f_{1}=224,6 \mathrm{~Hz}$

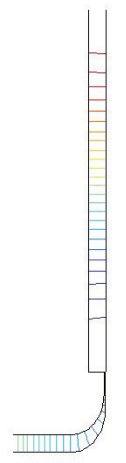

$f_{2}=307,0 \mathrm{~Hz}$

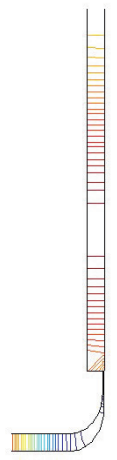

$f_{3}=406,1 \mathrm{~Hz}$

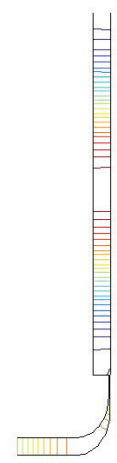

$f_{4}=482,5 \mathrm{~Hz}$

Fig. 7. Premiers modes propres de la cavité acoustique délimitée par le panier et la cuve.

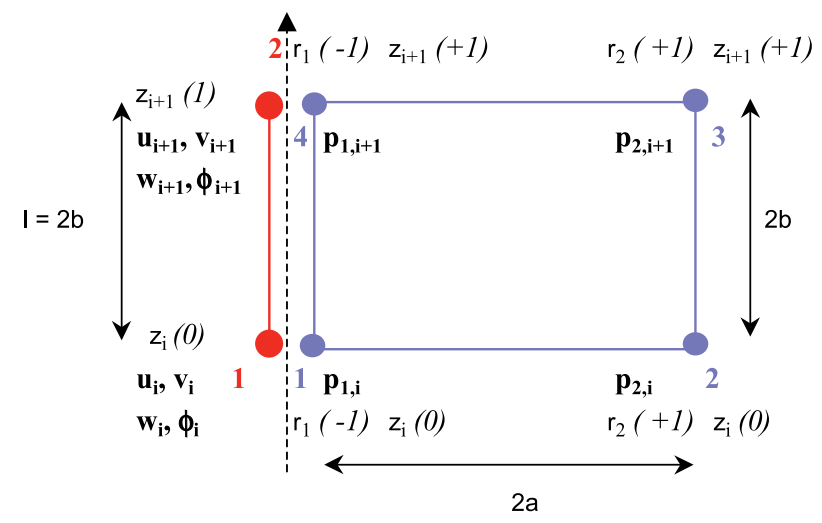

Fig. 8. Couplage éléments finis/éléments finis.

Le problème aux valeurs propres à résoudre dans le cas du couplage de la géométrie industrielle prend la forme générale suivante :

$$
\begin{aligned}
& {\left[\begin{array}{ccc}
\mathbf{K}_{\mathrm{C}} & \mathbf{0} & \mathbf{R}_{\mathrm{C}}^{\mathrm{T}} \\
\mathbf{0} & \mathbf{K}_{\mathrm{P}} & \mathbf{R}_{\mathrm{P}}^{\mathrm{T}} \\
\mathbf{0} & \mathbf{0} & \mathbf{K}_{\mathrm{F}}
\end{array}\right] \times\left\{\begin{array}{c}
\mathbf{U}_{\mathrm{C}}(\omega) \\
\mathbf{U}_{\mathrm{P}}(\omega) \\
\mathbf{P}(\omega)
\end{array}\right\}=} \\
& \omega^{2}\left[\begin{array}{ccc}
\mathbf{M}_{\mathrm{C}} & \mathbf{0} & \mathbf{0} \\
\mathbf{0} & \mathbf{M}_{\mathrm{P}} & \mathbf{0} \\
-\rho_{\mathrm{F}} \mathbf{R}_{\mathrm{C}} & -\rho_{\mathrm{F}} \mathbf{R}_{\mathrm{P}} & \mathbf{M}_{\mathrm{F}}
\end{array}\right] \times\left\{\begin{array}{c}
\mathbf{U}_{\mathrm{C}}(\omega) \\
\mathbf{U}_{\mathrm{P}}(\omega) \\
\mathbf{P}(\omega)
\end{array}\right\}
\end{aligned}
$$

avec $\mathbf{M}_{\mathrm{C}}, \mathbf{K}_{\mathrm{C}}$ les matrices masse et raideur de la cuve, $\mathbf{M}_{\mathrm{P}}, \mathbf{K}_{\mathrm{P}}$ les matrices masse et raideur du panier, $\mathbf{M}_{\mathrm{F}}$, $\mathbf{K}_{\mathrm{F}}$ les matrices du fluide et $\mathbf{R}_{\mathrm{C}}, \mathbf{R}_{\mathrm{P}}$ les matrices de couplage de la cuve et du panier avec le fluide. Les matrices masse et raideur du problème couplé sont creuses comme le montre la figure 9 (la représentation proposée met en évidence les éléments non nuls des matrices de masse et de raideur du système couplé).

Le système obtenu après couplage est non symétrique : nous utilisons alors une adaptation de l'algorithme de Lanczos $[23,24]$ pour traiter le problème de l'extraction des éléments propres du problème non symétrique (7).
Tableau 5. Validation de l'implantation de l'algorithme non symétrique de Lanczos dans le code de calcul développé dans Matlab. Comparaison sur l'exemple théorique proposé dans [24].

\begin{tabular}{rr}
\hline Valeurs propres théoriques & Valeurs propres calculées \\
\hline $0,10000+0,00000 i$ & $0,1000+0,0000 i$ \\
$4,00000 \pm 1,00000 i$ & $4,0000 \pm 1,0000 i$ \\
$2,40000 \pm 7,32000 i$ & $2,39974 \pm 7,31897 i$ \\
$7,00000+0,00000 i$ & $6,99999+0,00000 i$ \\
$7,00000+0,00000 i$ & $7,00088+0,00000 i$ \\
$8,00000 \pm 127,00000 i$ & $7,99997 \pm 127,00001 i$ \\
$11,00000+0,00000 i$ & $11,00005+0,00000 i$ \\
$130,000000+0,00000 i$ & $129,99998+0,00000 i$ \\
$-2000,00000+0,00000 i$ & $-1999,99995+0,00000 i$ \\
\hline
\end{tabular}

L'algorithme de Lanczos non symétrique est implanté dans le code de calcul développé dans Matlab, et validé par comparaison avec le cas théorique proposé dans [24] et rappelé en annexe; les résultats des deux calculs sont donnés dans le tableau 5 .

On conduit une analyse modale pour un problème couplant le fluide avec l'ensemble panier et cuve.

Le tableau 6 compare les valeurs des premières valeurs propres du système complet (panier et cuve) couplé avec le fluide dans le cas d'un calcul Matlab et d'un calcul Ansys. Le calcul de référence Ansys est obtenu par couplage éléments finis/éléments finis en formulation déplacement/déplacement et représentation des inconnues en série de Fourier, le calcul Matlab utilise le couplage éléments finis/éléments finis en formulation pression/déplacement et représentation des inconnues en série de Fourier, selon les principes exposés précédemment.

La figure 10 donne une représentation des déformées modales des premiers modes d'ordre $n=1$ du système couplé panier, cuve et fluide primaire.

Les résultats de calcul obtenus avec les deux méthodes sont proches, les écarts étant toutefois plus importants que pour le modèle de panier ou de cuve seuls (écarts de l'ordre de $4 \%$ en moyenne). L'écart devient assez significatif pour les fréquences au-delà du cinquième mode; 

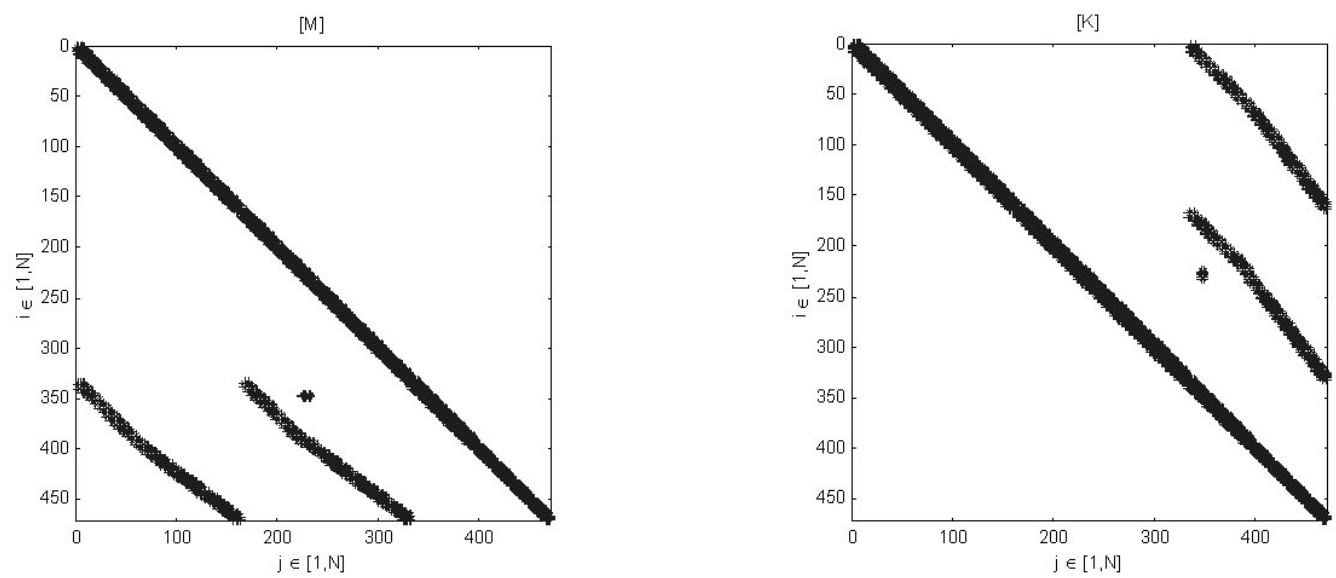

Fig. 9. Structure des matrices masse et raideur pour le problème couplé panier, cuve et fluide en formulation $(\mathbf{u}, p)$.

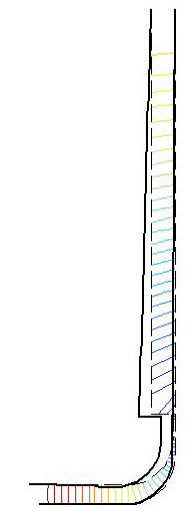

$f_{1}=26,03 \mathrm{~Hz}$

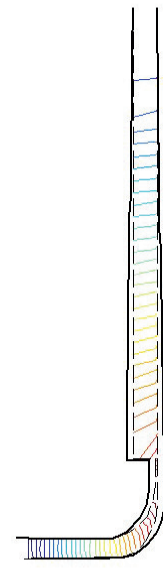

$f_{2}=65,52 \mathrm{~Hz}$

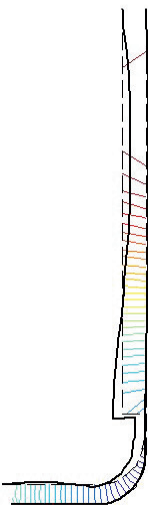

$f_{3}=102,94 \mathrm{~Hz}$

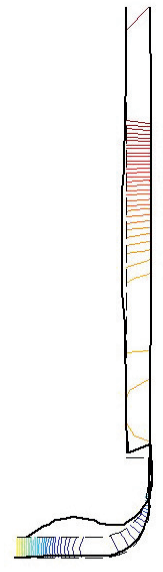

$f_{4}=162,30 \mathrm{~Hz}$

Fig. 10. Premiers modes propres de l'ensemble panier et cuve couplé avec le fluide.

Tableau 6. Première fréquences propres du système couplé. Comparaison des résultats de calcul Matlab et Anys.

\begin{tabular}{rrrr}
\hline Fréquence $(\mathrm{Hz})$ & Calcul Ansys & Calcul Matlab & $\varepsilon(\%)$ \\
\hline$f_{1}$ & 26,66 & 26,03 & $-2,36$ \\
$f_{2}$ & 64,02 & 65,52 & 2,50 \\
$f_{3}$ & 108,33 & 102,94 & $-4,98$ \\
$f_{4}$ & 158,24 & 162,30 & 2,57 \\
$f_{5}$ & 201,23 & 180,32 & 10,39 \\
\hline
\end{tabular}

nous atteignons les limites de l'outil Matlab pour le traitement de ce type de problèmes : la taille des problèmes devient trop importante pour pouvoir obtenir une bonne précision de calcul avec les fonctions de base de Matlab ${ }^{4}$. Néanmoins, les résultats donnés par le code Matlab restent très satisfaisants pour un calcul des premiers modes.

La figure 11 donne les courbes de convergence des trois premières fréquences propres du système couplé

\footnotetext{
${ }^{4}$ Une solution consisterait à développer certaines fonctions $\mathrm{du}$ programme sous forme de sous routines $\mathrm{C}++$ ou Fortran, compilées et intégrées au code Matlab [15].
}

en fonction de la taille $N$ du problème matriciel ( $\varepsilon$ est l'écart relatif entre les résultats du calcul Matlab et les résultats du calcul de référence avec Ansys en formulation déplacement/déplacement) et la figure 12 compare les temps de calcul pour l'extraction des valeurs propres du système couplé en utilisant un PC standard et une station Unix avec les mêmes versions de Matlab. L'utilisation de Matlab dans l'environnement Unix est nécessaire pour pouvoir traiter un problème de grande dimension (à partir de $N \approx 500)$.

\subsection{Approche « couplage fort », formulation symétrique}

La formulation du problème couplé utilisant la description $(\mathbf{u}, p)$ (déplacement/pression) conduit à un problème aux valeurs propres non symétriques :

$$
\left[\begin{array}{cc}
\mathbf{K}_{\mathrm{S}} & \mathbf{R}^{\mathrm{T}} \\
\mathbf{0} & \mathbf{K}_{\mathrm{F}}
\end{array}\right] \times\left\{\begin{array}{l}
\mathbf{U}(\omega) \\
\mathbf{P}(\omega)
\end{array}\right\}=\omega^{2}\left[\begin{array}{cc}
\mathbf{M}_{\mathrm{S}} & \mathbf{0} \\
-\rho_{\mathrm{F}} \mathbf{R}^{\mathrm{T}} & \mathbf{M}_{\mathrm{F}}
\end{array}\right] \times\left\{\begin{array}{l}
\mathbf{U}(\omega) \\
\mathbf{P}(\omega)
\end{array}\right\}
$$




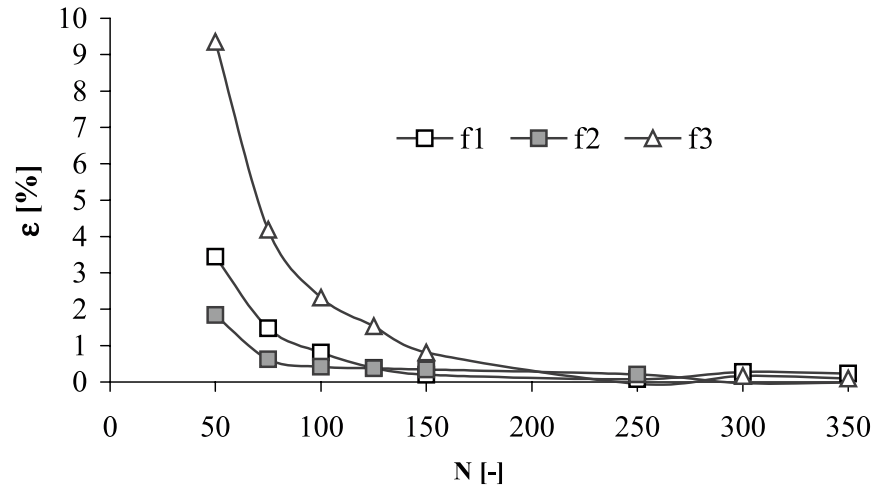

Fig. 11. Courbes de convergence des premières fréquences propres du panier et de la cuve couplés avec le fluide.

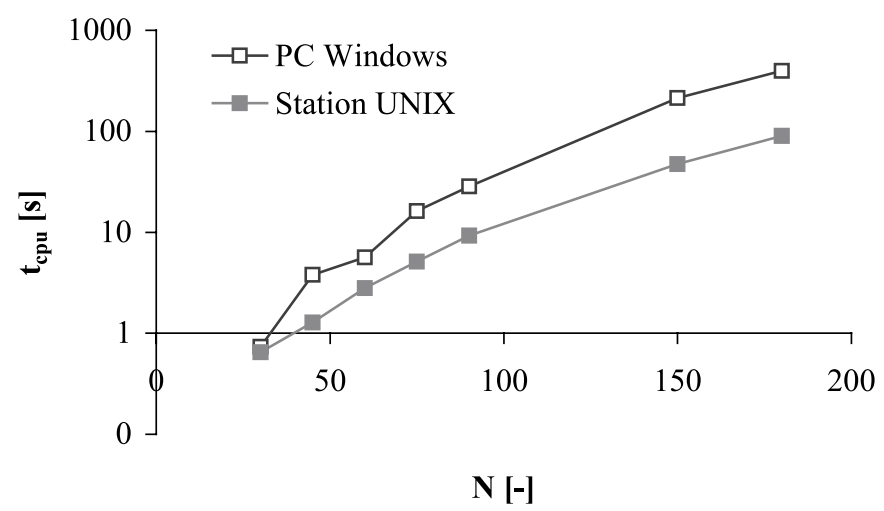

Fig. 12. Temps de calcul CPU pour l'extraction des valeurs propres du système couplé avec deux plateformes de calcul.

L'utilisation d'une formulation du problème couplé à l'aide d'une description $(\mathbf{u}, p, \varphi)$ (déplacement, pression, potentiel des déplacements [13]) permet d'obtenir un problème symétrique ${ }^{5}$. Les équations continues du problème couplé sont alors les suivantes. $\Omega_{\mathrm{S}}$ désignant le domaine structure, de frontières disjointes $\partial \Omega_{\mathrm{Sn}}$ (effort imposé), $\partial \Omega_{\mathrm{So}}$ (déplacement imposé) et $\Gamma_{\mathrm{FS}}$ (interface avec le fluide), les équations du problème structure sont alors :

$$
\begin{aligned}
\rho_{S} \omega^{2} u_{i}+\frac{\partial \sigma_{i j}(\mathbf{u})}{\partial x_{i j}}=0 \text { dans } \Omega_{\mathrm{S}} \\
\sigma_{i j}(\mathbf{u}) \cdot n_{j}^{\mathrm{S}}=0 \text { sur } \partial \Omega_{\mathrm{Sn}} \\
u_{i}=0 \text { sur } \partial \Omega_{\mathrm{So}}
\end{aligned}
$$

$\Omega_{\mathrm{F}}$ désignant le domaine fluide, de frontières $\partial \Omega_{\mathrm{Fn}}$ (déplacement imposé), $\partial \Omega_{\mathrm{Fo}}$ (pression imposée) et $\Gamma_{\mathrm{SF}}$

\footnotetext{
${ }^{5}$ D'autres formulations symétriques sont possibles, voir par exemple $[25,26]$.
}

(interface avec la structure), les équations du problème fluide sont alors :

$$
\begin{aligned}
\rho_{\mathrm{F}} \frac{\partial^{2} \varphi}{\partial x_{i} \partial x_{j}}+\frac{p}{c^{2}} & =0 \text { dans } \Omega_{\mathrm{F}} \\
\frac{p}{\rho_{\mathrm{F}} c^{2}} & =\frac{\omega^{2}}{c^{2}} \varphi \text { dans } \Omega_{\mathrm{F}} \\
\frac{\partial \varphi}{\partial x_{j}} \cdot n_{j} & =0 \text { sur } \partial \Omega_{\mathrm{Fn}} \\
p & =0 \text { sur } \partial \Omega_{\mathrm{Fo}}
\end{aligned}
$$

Les équations de couplage entre les deux problèmes expriment la continuité de la composante normale du tenseur des contraintes et la continuité de la composante normale du déplacement :

$$
\begin{array}{r}
\sigma_{i j}(\mathbf{u}) \cdot n_{j}=\varphi \rho_{\mathrm{F}} \omega^{2} n_{i} \text { sur } \Gamma_{\mathrm{FS}} \\
\frac{\partial \varphi}{\partial x_{j}} \cdot n_{j}=u_{j} \cdot n_{j} \text { sur } \Gamma_{\mathrm{SF}}
\end{array}
$$

La formulation variationnelle $\mathrm{du}$ problème spectral ainsi posé est alors la suivante : trouver $\mathbf{u}(\omega), p(\omega)$, $\varphi(\omega)$ dans $V_{\mathrm{S}} \times V_{\mathrm{F}} \times H_{\mathrm{F}}$ et $\omega$ réel tels que pour tout $\delta \mathbf{u}$, $\delta p, \delta \varphi$ dans $V_{\mathrm{S}} \times V_{\mathrm{F}} \times H_{\mathrm{F}}$, on ait [27-29]:

$$
\begin{aligned}
& k_{\mathrm{S}}(\mathbf{u}(\omega), \delta \mathbf{u})+k_{\mathrm{F}}^{\prime}(p(\omega), \delta p)= \\
& \quad \omega^{2} \times\left[m_{\mathrm{S}}(\mathbf{u}(\omega), \delta \mathbf{u})-m_{\mathrm{F}}^{\prime}(\varphi(\omega), \delta \varphi)+\tilde{r}(\mathbf{u}(\omega), \delta \varphi)\right. \\
& \left.\quad+\tilde{r}^{*}(\varphi(\omega), \delta \mathbf{u})+\tilde{m}_{\mathrm{F}}(p(\omega), \delta \varphi)+\tilde{m}_{\mathrm{F}}^{*}(\varphi(\omega), \delta p)\right]
\end{aligned}
$$

Les espaces fonctionnels sont respectivement [30] : $V_{\mathrm{S}}=\left\{\mathbf{u} \in H^{1}\left(\Omega_{\mathrm{S}}\right)^{3},\left.\mathbf{u}\right|_{\partial \Omega_{\mathrm{So}}}=0\right\}, V_{\mathrm{F}}=\left\{\varphi \in H^{1}\left(\Omega_{\mathrm{F}}\right)\right\}$ et $H_{\mathrm{F}}=\left\{p \in L^{2}\left(\Omega_{\mathrm{F}}\right),\left.p\right|_{\partial \Omega_{\mathrm{Fo}}}=0\right\}$; les formes bilinéaires définies sur ces espaces sont données par $k_{\mathrm{S}}(\mathbf{u}, \delta \mathbf{u})=\int_{\Omega_{\mathrm{S}}} \sigma_{i j}(\mathbf{u}) \cdot \varepsilon_{i j}(\delta \mathbf{u}) \mathrm{d} \Omega, m_{\mathrm{S}}(\mathbf{u}, \delta \mathbf{u})=\int_{\Omega_{\mathrm{S}}} \rho_{\mathrm{S}} \mathbf{u} \cdot$ $\delta \mathbf{u d} \Omega, m_{\mathrm{F}}(p, \delta p)=\int_{\Omega_{\mathrm{F}}} \frac{p \delta p}{c^{2}} \mathrm{~d} \Omega \quad k_{\mathrm{F}}^{\prime}(p, \delta p)=\int_{\Omega_{\mathrm{F}}} \frac{p \delta p}{\rho_{\mathrm{F}} c^{2}} \mathrm{~d} \Omega$, $\tilde{m}_{\mathrm{F}}(p, \delta \varphi)=\int_{\Omega_{\mathrm{F}}} \frac{p \delta \varphi}{c^{2}} \mathrm{~d} \Omega, \tilde{r}(u, \delta \varphi)=\int_{\Gamma} \rho_{\mathrm{F}} \mathbf{u} \cdot \mathbf{n} \delta \varphi \mathrm{d} \Gamma$ et $\tilde{m}_{\mathrm{F}}(p, \delta \varphi)=\int_{\Omega_{\mathrm{F}}} \frac{p \delta \varphi}{c^{2}} \mathrm{~d} \Omega ; \tilde{m}_{\mathrm{F}}^{*}$ et $\tilde{r} *$ sont les opérateurs transposés de $\tilde{m}_{\mathrm{F}}$ et $\tilde{r}$.

La discrétisation de la formulation variationnelle (11) conduit au problème aux valeurs propres suivant :

$$
\left[\begin{array}{ccc}
\mathbf{K}_{\mathrm{S}} & \mathbf{0} & \mathbf{0} \\
\mathbf{0} & \mathbf{K}_{\mathrm{F}}^{\prime} & \mathbf{0} \\
\mathbf{0} & \mathbf{0} & \mathbf{0}
\end{array}\right] \times\left\{\begin{array}{l}
\mathbf{U}(\omega) \\
\mathbf{P}(\omega) \\
\boldsymbol{\Phi}(\omega)
\end{array}\right\}=
$$

$$
\omega^{2}\left[\begin{array}{ccc}
\mathbf{M}_{\mathrm{S}} & \mathbf{0} & \tilde{\mathbf{R}} \\
\mathbf{0} & \mathbf{0} & \tilde{\mathbf{M}}_{\mathrm{F}} \\
\tilde{\mathbf{R}}^{\mathrm{T}} & \tilde{\mathbf{M}}_{\mathrm{F}}^{\mathrm{T}} & -\mathbf{M}_{\mathrm{F}}^{\prime}
\end{array}\right] \times\left\{\begin{array}{c}
\mathbf{U}(\omega) \\
\mathbf{P}(\omega) \\
\boldsymbol{\Phi}(\omega)
\end{array}\right\}
$$



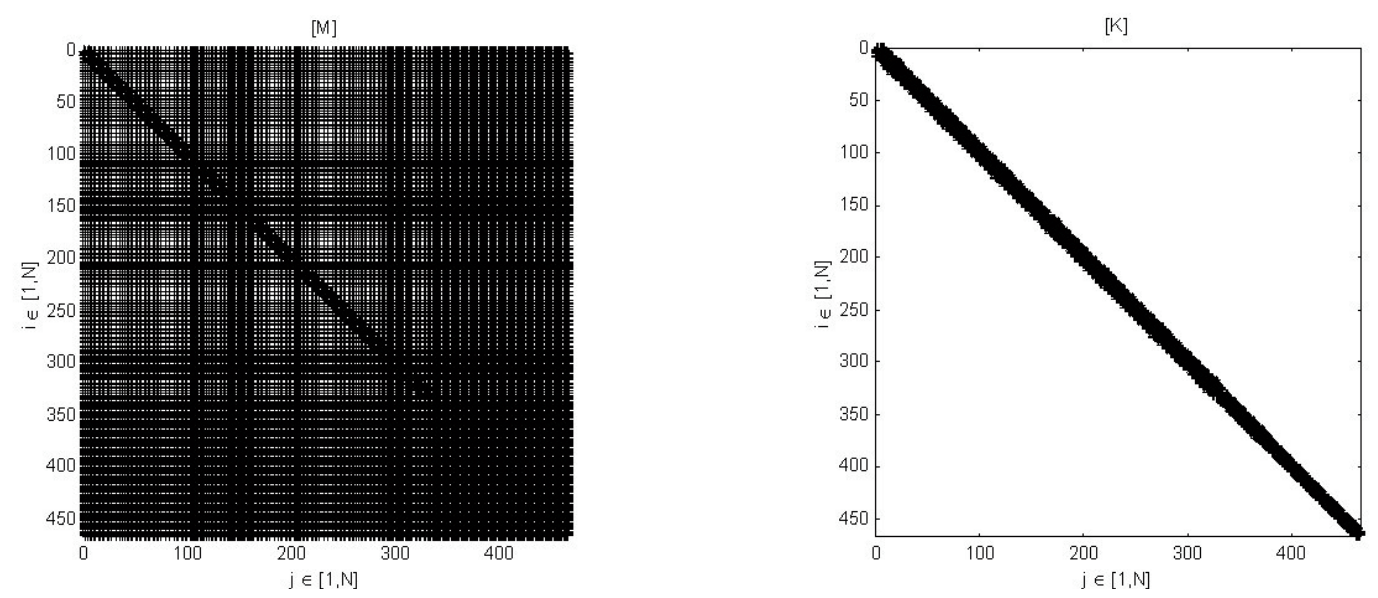

Fig. 13. Structure des matrices masse et raideur pour le problème couplé panier, cuve et fluide en formulation $(\mathbf{u}, p, \varphi)$.

Tableau 7. Premières fréquences propres du problème couplé, modes d'ordre $n=1$. Comparaison des formulations $(\mathbf{u}, p)$ et $(\mathbf{u}, p, \varphi)$.

\begin{tabular}{rrrr}
\hline Fréquence $(\mathrm{Hz})$ & $\begin{array}{r}\text { Formulation } \\
(\mathbf{u}, p)\end{array}$ & $\begin{array}{r}\text { Formulation } \\
(\mathbf{u}, p, \varphi)\end{array}$ & $\varepsilon(\%)$ \\
\hline$f_{1}$ & 26,03 & 25,99 & 0,15 \\
$f_{2}$ & 65,62 & 64,72 & 1,37 \\
$f_{3}$ & 102,94 & 103,98 & $-1,01$ \\
$f_{4}$ & 162,30 & 162,17 & 0,08 \\
$f_{5}$ & 180,32 & 183,70 & $-1,87$ \\
\hline
\end{tabular}

Après élimination de la variable $\Phi$, le problème aux valeurs propres (12) est écrit sous la forme symétrique suivante :

$$
\begin{aligned}
& {\left[\begin{array}{cc}
\mathbf{K}_{\mathrm{S}} & \mathbf{0} \\
\mathbf{0} & \mathbf{K}_{\mathrm{F}}^{\prime}
\end{array}\right] \times\left\{\begin{array}{l}
\mathbf{U}(\omega) \\
\mathbf{P}(\omega)
\end{array}\right\}=} \\
& \quad \omega^{2}\left[\begin{array}{cc}
\mathbf{M}_{\mathrm{S}}+\tilde{\mathbf{R}} \mathbf{M}_{\mathrm{F}}^{\prime-1} \tilde{\mathbf{R}}^{\mathrm{T}} & \tilde{\mathbf{R}}_{\mathrm{F}}^{\prime-1} \tilde{\mathbf{M}}_{\mathrm{F}}^{\mathrm{T}} \\
\tilde{\mathbf{M}}_{\mathrm{F}} \mathbf{M}_{\mathrm{F}}^{\prime-1} \tilde{\mathbf{R}}^{\mathrm{T}} & \tilde{\mathbf{M}}_{\mathrm{F}} \mathbf{M}_{\mathrm{F}}^{\prime-1} \tilde{\mathbf{M}}_{\mathrm{F}}^{\mathrm{T}}
\end{array}\right] \times\left\{\begin{array}{c}
\mathbf{U}(\omega) \\
\mathbf{P}(\omega)
\end{array}\right\}
\end{aligned}
$$

En utilisant les mêmes fonctions de forme pour $p$ et $\varphi$, en supposant les caractéristiques fluide $c$ et $\rho_{\mathrm{F}}$ constantes, nous avons :

$$
\mathbf{K}_{\mathrm{F}}^{\prime}=\frac{\mathbf{M}_{\mathrm{F}}}{\rho_{\mathrm{F}}} \quad \tilde{\mathbf{R}}=\rho_{\mathrm{F}} \mathbf{R} \quad \mathbf{M}_{\mathrm{F}}^{\prime}=\rho_{\mathrm{F}} \mathbf{K}_{\mathrm{F}} \quad \mathbf{M}_{\mathrm{F}}^{\prime}=\mathbf{M}_{\mathrm{F}}
$$

Le problème (13) est symétrique mais la matrice de masse n'est plus creuse, comme le montre la figure 13 (représentation des termes non nuls des matrices).

Le tableau 7 compare les résultats de calculs obtenus avec le code Matlab en utilisant les formulations $(\mathbf{u}, p)$ et $(\mathbf{u}, p, \varphi)$. Les écarts entre les deux types de calcul sont de l'ordre de $1 \%$. Les modes propres obtenus à partir de la formulation $(\mathbf{u}, p, \varphi)$ possèdent les propriétés d'orthogonalité vis-à-vis des matrices masse et raideur du système couplé, ce qui permet l'application de méthodes modales pour les calculs dynamiques [31].

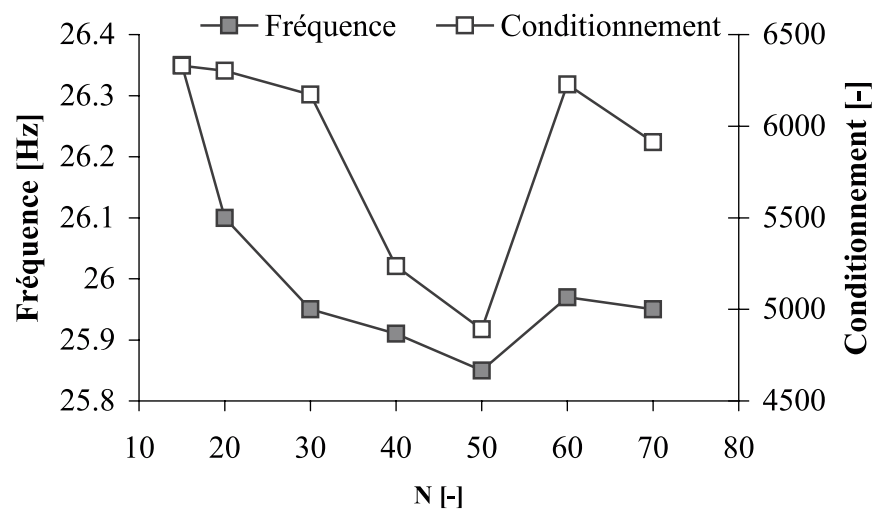

Fig. 14. Premières fréquence propre du système couplé en formulation $(\mathbf{u}, p, \varphi)$ et conditionnement en norme $\|-\|_{1}$ de la matrice $\mathbf{K}_{\mathrm{F}}$ en fonction du nombre de degrés de liberté $N$ du problème.

La formulation $(\mathbf{u}, p, \varphi)$ fait intervenir la matrice $\mathbf{K}_{\mathrm{F}}^{-1}$ : le calcul des valeurs propres du système couplé est donc influencé par la précision de l'inversion matricielle. Dans Matlab, nous utilisons la fonction inv, qui réalise une inversion matricielle à partir de l'algorithme de Cholesky ou l'algorithme de Gauss selon la nature définie positive de la matrice à inverser [20]. La figure 14 donne l'évolution de la première fréquence propre du système couplé en fonction de la taille du problème et l'évolution du conditionnement de la matrice $\mathbf{K}_{\mathrm{F}}$ pour le problème de l'inversion matricielle et met en évidence la corrélation entre les deux évolutions.

Si le fluide est considéré incompressible, $c \rightarrow+\infty$ et $m_{\mathrm{F}}(.,.) \equiv 0$. Le problème couplé se réduit du point de vue structure au problème symétrique suivant, faisant intervenir l'opérateur de masse ajoutée [13] :

$$
\omega^{2}\left(\mathbf{M}_{\mathrm{S}}+\mathbf{M}_{\mathrm{H}}\right) \times \mathbf{U}(\omega)=\mathbf{K}_{\mathrm{S}} \times \mathbf{U}(\omega)
$$

avec :

$$
\mathbf{M}_{\mathrm{H}}=\rho_{\mathrm{F}} \mathbf{R}^{\mathrm{T}} \mathbf{K}_{\mathrm{F}}^{-1} \mathbf{R}
$$

L'opérateur $\mathbf{M}_{\mathrm{H}}$ étant symétrique, le problème aux valeurs propres (15) l'est également. 
Tableau 8. Premières fréquences propres du problème couplé. Comparaison entre un calcul couplé compressible et incompressible.

\begin{tabular}{rrr}
\hline Fréquence $(\mathrm{Hz})$ & $\begin{array}{r}\text { Fluide } \\
\text { compressible }\end{array}$ & $\begin{array}{c}\text { Fluide } \\
\text { incompressible }\end{array}$ \\
\hline$f_{1}$ & 26,03 & 25,99 \\
$f_{2}$ & 65,62 & 64,82 \\
$f_{3}$ & 102,94 & 104,23 \\
$f_{4}$ & 162,30 & 179,25 \\
\hline
\end{tabular}

Tableau 9. Calculs de la première fréquence propre pour différentes situations de calcul (panier seul, cuve seule, fluide seul, système couplé). Formulation fluide en pression, formulation structure en déplacement.

\begin{tabular}{rrrr}
\hline Modèle & $\begin{array}{r}\text { Calcul } \\
\text { Ansys 3D }\end{array}$ & $\begin{array}{r}\text { Calcul } \\
\text { Matlab 2D }\end{array}$ & $\varepsilon(\%)$ \\
\hline Panier & 41,25 & 44,70 & 8,36 \\
Cuve & 78,64 & 78,70 & 0,08 \\
Fluide & 224,68 & 224,68 & $-0,05$ \\
Panier, cuve et fluide & 25,94 & 26,03 & 0,35 \\
\hline
\end{tabular}

Le tableau 8 donne les fréquences propres du système couplé calculé dans le cas d'un fluide incompressible et d'un fluide compressible : les fréquences obtenues et les déformées modales du système structure sont identiques dans les deux cas, ce qui montre que les effets de compressibilité n'interviennent pas sur les premiers modes du système couplé. Les fréquences propres du système couplé avec fluide incompressible sont suffisamment éloignées des premières fréquences propres de la cavité acoustique, ce qui permet de négliger les effets acoustiques pour les premiers modes [31].

Nous comparons enfin les résultats de calcul obtenus avec un modèle tridimensionnel Ansys utilisant les éléments structure shell63 (éléments coques linéaires bidimensionnels [16]) et les éléments fluide fluid30 (éléments fluides acoustiques linéaires tridimensionnels [21]) et un modèle bidimensionnel axisymétrique utilisant le code de calcul Matlab. Le tableau 9 donne une comparaison des valeurs du premier mode calculé pour le panier, la cuve, le fluide seuls ou couplés. Les résultats de calcul sont équivalents dans les deux approches, mais les temps de calcul sont très importants dans le cas d'un modèle tridimensionnel, particulièrement pour le système couplé compte tenu de la non symétrie du problème aux valeurs propres.

\section{Conclusion}

La présente étude a porté sur l'analyse modale d'une structure industrielle, en prenant en compte le couplage fluide/structure. Une simplification de la géométrie du problème réel nous a permis de nous placer dans le cas d'un problème à géométrie de révolution, présentant un chargement non axisymétrique.
La discrétisation du problème couplé a mis en œuvre des éléments finis axisymétriques développés en série de Fourier :

- élément fini structure : coque élastique formulé en déplacement;

- élément fini fluide : fluide acoustique formulé en pression.

Un code de calcul dédié a été programmé sous Matlab et réalise le maillage du problème, l'assemblage des matrices élémentaires, la détermination des éléments propres du système et la visualisation des modes propres.

Les comparaisons entre les résultats de calcul Matlab et Ansys (utilisant un couplage déplacement/déplacement) ont permis de valider les développements réalisés dans Matlab et la capacité du code élémentaire à traiter des géométries simples mais représentatives de cas industriels.

À terme, l'élément fluide ainsi développé et son couplage avec des éléments structure sera implanté dans le code Ansys pour permettre une analyse de géométries complexes, en utilisant la formulation pression/déplacement dans le cas de géométries de révolution avec termes de chargement non axisymétriques.

\section{Annexe : Cas élémentaire de validation du programme de calcul des modes propres d'un problème généralisé non symétrique}

Le programme d'extraction des valeurs propres pour un problème généralisé non symétrique utilisé dans le code développé dans Matlab est une adaptation de l'algorithme de Lanczos [23]. Le principe de l'algorithme est détaillé dans [24]; cet algorithme est implanté dans le code de calcul Matlab pour traiter le cas industriel présenté, la validation de la fonction Matlab ainsi obtenue est faite à partir du problème présenté dans [24], correspondant aux matrices $12 \times 12$ suivantes :

$[\mathbf{M}]=\left|\begin{array}{rrrrrrrrrrrr}-410 & 16 & 90 & 33 & 2 & 88 & 125 & -215 & -16 & 43 & 64 & 7 \\ 340 & 38 & 99 & 81 & 2 & 54 & 195 & 220 & -41 & -1 & -92 & -26 \\ -40 & -84 & -114 & 60 & -45 & 82 & -45 & 145 & 2 & 25 & 52 & 3 \\ -110 & -30 & 75 & -120 & -40 & 26 & -220 & 150 & 44 & -32 & 24 & -15 \\ 190 & -76 & 63 & 21 & 16 & -34 & -65 & 25 & 6 & -8 & -12 & -38 \\ 30 & 4 & 27 & -33 & 31 & 74 & 160 & -170 & -46 & -15 & 64 & 49 \\ 110 & 96 & -129 & -132 & 8 & 66 & -125 & 0 & 4 & 43 & 78 & -4 \\ 340 & 26 & -39 & -144 & -43 & -92 & -30 & -145 & -36 & 19 & -68 & 6 \\ -490 & -68 & -141 & -12 & -3 & 48 & 0 & 180 & 20 & 48 & 74 & -48 \\ -30 & 54 & -12 & 9 & -39 & 22 & 160 & 40 & -11 & 12 & 82 & -45 \\ -170 & 10 & 102 & -147 & 13 & -20 & -195 & 75 & -27 & -19 & 86 & 12 \\ 290 & -4 & -54 & 147 & -29 & 50 & 140 & -195 & -17 & 40 & 44 & 18\end{array}\right|$




\section{Références}

[1] J. Makerle, Finite and Boundary Element Techniques in Acoustics, a Bibliography (1990-1992), Finite Element Analysis Design 15 (1994) 263-272

[2] J. Makerle, Fluid-Structure Interaction Problems, Finite Element Approach and Boundary Elements Approaches, A Bibliography, Finite Elements in Analysis and Design 31 (1999) 231-240

[3] G. Delhommeau, Contribution à l'étude théorique et à la résolution numérique de problème de résistance de vagues, Thèse de Doctorat, École nationale supérieure de mécanique de Nantes, 1978

[4] G. Delhommeau, Les problèmes de diffraction-radiation et de résistance de vague : étude théorique et résolution numérique par la méthode des singularités, Thèse de doctorat d'État, École nationale supérieure de mécanique de Nantes, 1987

[5] H. Oudin, Étude du comportement hydroélastique de structures marines par une formulation mixte équations intégrale-éléments finis, Thèse de doctorat, École nationale supérieure de mécanique de Nantes, 1986

[6] B. Peseux, Contribution à l'étude de structures partiellement ou totalement immergées en matériau homogène ou en composite, Thèse de doctorat d'État, École nationale supérieure de mécaniques de Nantes, 1989

[7] B. Peseux, G. Delhommeau, Fluid-Structure Interaction: Influence of Compressibility on the Dynamic Response of Immersed Structures, Aéronautique Conférence DGLR/AIAA, Strasbourg, 1993

[8] B. Peseux, F. Bérot, Vibro-Acoustic Behavior of Submersed Cylindrical Shells: Analytical Formulation and Numerical Model, J. Fluids and Structures 12 (1998) 959-1003

[9] B. Peseux, A. Piard, Dynamic Response of Submersed Structures in Bonded Domain with Gravity Waves, Pressure Vessel and Piping, San Diego, 26-30 July 1998, 336, pp. 187-196

[10] J.P. Quevat, Couplage de la méthode des équations intégrales et de la méthode des éléments finis pour la résolution d'un problème d'hydroélasticité, Thèse de doctorat, École nationale supérieure de mécanique de Nantes, 1979

[11] J. Makerle, Finite Element in the Analysis of Pressure Vessel and Piping, a Bibliography (19761996), Int. J. Pressure Vessel and Piping 69 (1996) 279-339

[12] J. Makerle, Earthquake Analysis of Structures: FEM and BEM Approaches, a Bibliography (1995-1998), Finite Element Analysis Design 32 (1999) 113-124

[13] H.J.P. Morand, R. Ohayon, Fluid Structure Interaction, Wiley \& Sons, 1995
[14] O.C. Zienkiewicz, R.L. Taylor, The Finite Element Method, Basic Formulation and Linear Problems, 4th edition, Mac Graw Hill, 1989

[15] M. Mokhtari, A. Mesbah, Apprendre et maîtriser Matlab, Springer Verlag, 1997

[16] P. Khonke, ANSYS Theory Reference, Swanson Analysis System, 1986

[17] J.F. Sigrist, C. Lainé, B. Peseux, Calcul couplé fluide/structure avec Ansys : étude numérique et expérimentale d'une plaque élastique en contact avec un fluide lourd compressible, J. Physique France 12 (2002) Pr11-137-144

[18] D.G. Ashwell, R.H. Gallagher, Finite Elements for Thin Shells and Curved Members, Wiley, 1976

[19] P.G. Ciarlet, Introduction à l'analyse numérique matricielle et à l'optimisation, Masson, 1988

[20] G.H. Golub, C.F. Van Loan, Matrix Computation, Johns Hopkins University Press, 1984

[21] D.B. Woyak, Acoustic and Fluid Structure Interaction, Swanson Analysis System, 1995

[22] J.F. Sigrist, C. Lainé, D. Lemoine, B. Peseux, Choice and limits of a linear fluid model for the numerical study in dynamic fluid structure interaction problem, Pressure Vessel and Piping, Cleveland, 20-24 July 2003, 454, pp. 87-93

[23] F. Chatelin, Valeurs propres de matrices, Masson, 1988

[24] C. Rajakumar, C.G. Rogers, The Lanczos Algorithm Applied to Unsymmetric Generalized Eigenvalue Problem, Int. J. Numerical Methods in Engineering 32 (1991) 1009-1026

[25] G.C. Everstine, Letter to the Editor: A Symmetric Potential Formulation for Fluid-Structure Interaction, J. Sound and Vibration 79 (1981) 157-190

[26] G. Sandberg, P.A. Göransson, A Symmetric Finite Element Formulation for Acoustic Fluid-Structure Interaction Analysis, J. Sound and Vibration 123 (1988) 507-515

[27] J. Boujot, Interaction fluide structure en régime transitoire, La Recherche Aérospatiale 3 (1984) 203-209

[28] J. Boujot, Étude d'une famille de problèmes d'évolution du second ordre correspondant à divers modèles de couplage fluide structure, Compte Rendu à l'Académie des Sciences, I, 301 (1985) 391-394

[29] J. Boujot, Mathematical Formulation of Fluid Structure Interaction Problems, Mathematical Modelling and Numerical Analysis 21 (1987) 239-260

[30] P.A. Raviart, J.M. Thomas, Introduction à l'analyse numérique des équations aux dérivées partielles, Masson, 1983

[31] R.J. Gibert, Vibration des structures, Interaction avec les fluides, Sources d'excitation aléatoires, Collection de la Direction des Études et Recherches d'Électricité de France, 69 (1986) 